\title{
The Classical Limit of the Relativistic Vlasov-Maxwell System
}

\section{Jack Schaeffer}

Department of Mathematics, Carnegie-Mellon University, Pittsburgh, Pennsylvania 15213, USA

\begin{abstract}
Solutions of the relativistic Vlasov-Maxwell system of partial differential equations are considered in three space dimensions. The speed of light, $c$, appears as a parameter in this system. For smooth Cauchy data, classical solutions are shown to exist on a time interval that is independent of $c$. Then, using an integral representation for the electric and magnetic fields due to Glassey and Strauss [6], conditions are given under which solutions of the relativistic Vlasov-Maxwell system converge in a pointwise sense to solutions of the non-relativistic Vlasov-Poisson system at the asymptotic rate of $1 / c$, as $c$ tends to infinity.
\end{abstract}

\section{Introduction}

Consider the Cauchy problem

$(\mathrm{RVM})$

$$
\begin{cases}\partial_{t} f+\hat{v} \cdot \nabla_{x} f+\left(E+c^{-1} \hat{v} \times B\right) \cdot \nabla_{v} f=0 \\ \partial_{t} E=c \nabla \times B-4 \pi j & \nabla \cdot E=4 \pi \rho \\ \partial_{t} B=-c \nabla \times E & \nabla \cdot B=0 \\ \rho(x, t)=\int f(x, v, t) d v & j(x, t)=\int f(x, v, t) \hat{v} d v\end{cases}
$$

where

$$
\hat{v}=\left(1+c^{-2} v^{2}\right)^{-1 / 2} v
$$

and the initial conditions are

$$
\left\{\begin{array}{l}
f(x, v, 0)=f_{0}(x, v), \\
E(x, 0)=E_{0}(x) \\
B(x, 0)=B_{0}(x) .
\end{array}\right.
$$

Here $x$ and $v$ are points in $\mathbb{R}^{3}$ representing position and momentum respectively. $f$ gives the number density of a collisionless plasma consisting of a single species of charged particle acting under its selfinduced Lorentz force, $E+c^{-1} \hat{v} \times B$. The parameter $c$ is the speed of light. (RVM) is a relativistic version of the classical Vlasov-Maxwell system (VM), which may be obtained from (RVM) by replacing $\hat{v}$ with $v$. The formulation of the relativistic version is discussed in [11 and 13]. 
Our goal is to establish conditions under which solutions of (RVM) converge, as $c$ tends to infinity, to solutions of

$$
\left\{\begin{array}{l}
\partial_{t} f^{\infty}+v \cdot \nabla_{x} f^{\infty}+E^{\infty} \cdot \nabla_{v} f^{\infty}=0 \\
E^{\infty}(x, t)=\int \rho^{\infty}(y, t)|x-y|^{-3}(x-y) d y \\
\rho^{\infty}(x, t)=\int f^{\infty}(x, v, t) d v
\end{array}\right.
$$

with the initial condition

$$
f^{\infty}(x, v, 0)=f_{0}(x, v) .
$$

One purpose of this goal is to make precise the statement that relativistic effects are negligible if the speed of light is much greater than the velocities which occur. An additional advantage is that (VP) is more fully understood than (RVM), so we would be able to approximate a harder problem by an easier one.

Global weak solutions are known to exist for (VP) ([1 and 9]). Global classical solutions for (VP) have been shown to exist for symmetric data $([4,8$, and 14]) and for small data [3], but the problem of global existence of smooth solutions for unrestricted data is still open.

Local existence of smooth solutions of (VM) has been established by Wollman [15]. Glassey and Strauss [6] have shown for (RVM) that an a priori bound on momenta (i.e. there exists a continuous function $\beta:[0, T) \rightarrow[0, \infty)$ such that $|v|>\beta(t)$ implies $f(x, v, t)=0$ ) implies existence of a smooth solution for $0 \leqq t<T$. This theorem is analogous to Theorem 1 of [4].

The main results of this paper are the following two theorems:

Theorem 1. Assume that $f_{0} \in C^{1}\left(\mathbb{R}^{6}\right)$ is nonnegative and has compact support. Assume that $E_{0}$ and $B_{0}$ are in $C^{2}\left(\mathbb{R}^{3}\right) \cap W^{1, \infty}\left(\mathbb{R}^{3}\right) \cap W^{2,1}\left(\mathbb{R}^{3}\right)$ and satisfy

$$
\begin{aligned}
& \nabla \cdot E_{0}=4 \pi \int f_{0} d v, \\
& \nabla \cdot B_{0}=0 .
\end{aligned}
$$

Then there exists $T>0$ (independent of c) such that for each $c \geqq 1,(\mathrm{RVM})$ with the initial condition (RIC) has a unique $C^{1}$ solution $\left(f^{c}, E^{c}, B^{c}\right)$ on the time interval $[0, T)$. Furthermore there exist nondecreasing functions (independent of $c$ ) $P:[0, T) \rightarrow \mathbb{R}$ and $K:[0, T) \rightarrow \mathbb{R}$ such that

$$
\begin{array}{r}
f^{c}(x, v, t)=0 \quad \text { if } \quad|v| \geqq P(t), \\
\left|E^{c}(x, t)\right|+\left|B^{c}(x, t)\right| \leqq K(t),
\end{array}
$$

for all $t \in[0, T), x \in \mathbb{R}^{3}$, and $c \geqq 1$.

Theorem 2. Let $f_{0}$ be a nonnegative $C^{1}$ function of compact support in $\mathbb{R}^{6}$ and let

$$
E_{0}(x)=\iint f_{0}(x, v) d v|x-y|^{-3}(x-y) d y .
$$

Let $\left(f^{\infty}, E^{\infty}\right)$ be the unique $C^{1}$ solution of $(\mathrm{VP})$ with $f(x, v, 0)=f_{0}(x, v)$ that is known to exist by $[10]$ for some time $T_{1}>0$. Assume both of the following:

A. There exists $T_{2}>0$ such that for each $c \geqq 1$, (RVM) has a $C^{1}$ solution $\left(f^{c}, E^{c}, B^{c}\right)$ on $\left[0, T_{2}\right)$ with $f^{c}(x, v, 0)=f_{0}(x, v), E^{c}(x, 0)=E_{0}(x)$, and $B^{c}(x, 0)=0$; 
B. There exists a nondecreasing function $P:\left[0, T_{2}\right) \rightarrow \mathbb{R}$ such that

$$
f^{c}(x, v, t)=0 \text { if }|v| \geqq P(t)
$$

for all $x \in \mathbb{R}^{3}, t \in\left[0, T_{2}\right)$, and $c \geqq 1$.

Let $T=\min \left(T_{1}, T_{2}\right)$, then for every $T^{\prime} \in[0, T)$ there exists a constant $D$ (depending on $T^{\prime}$ and the data, but not on $\left.c\right)$ such that

$$
\left|f^{c}(x, v, t)-f^{\infty}(x, v, t)\right|+\left|E^{c}(x, t)-E^{\infty}(x, t)\right|+\left|B^{c}(x, t)\right| \leqq D c^{-1},
$$

for all $x \in \mathbb{R}^{3}, v \in \mathbb{R}^{3}, t \in\left[0, T^{\prime}\right]$, and $c \geqq 1$.

Comment. If we also assume that $f_{0}$ is $C^{2}$ then the hypotheses of Theorem 1 are satisfied with $B_{0}=0$. Hence if $f_{0}$ is $C^{2}$ then by Theorem 1 we know hypotheses $\mathrm{A}$ and B of Theorem 2 are satisfied for some $T_{2}>0$ and $P:\left[0, T_{2}\right) \rightarrow \mathbb{R}$.

As this work was being completed we learned that Degond [5] and independently Asano and Ukai [2] have recently established results similar to the above theorems for the classical system (VM). Here we deal with the relativistic system (RVM) which, in light of a recent blow up result, [7], for a relativistic version of (VP), may be significantly different from (VM). Also the analysis in this work differs from that in [5 and 2] in that it is based on an integral representation of the fields due to Glassey and Strauss [6]. Hence the integral representation of $E^{c}$ is compared directly with the Coulomb force,

$$
E^{\infty}(x, t)=\iint f^{\infty}(y, v, t)|x-y|^{-3}(x-y) d v d y,
$$

which holds for (VP) and the representation of $B^{c}$ is compared with zero. Pointwise estimates are obtained. It is hoped that this results in a more explicit understanding of how the two problems ((RVM) and (VP)) compare.

The following notation is used. Given $x$ and $y$ let

$$
\omega=|x-y|^{-1}(y-x) \text {. }
$$

$d \omega$ denotes the surface measure of the unit sphere, $d S$ denotes surface measure more generally. We assume in both Theorems 1 and 2 that $f_{0}$ has compact support, so let

$$
S_{0}=\sup \left\{|x| \text { : there exists } v \in \mathbb{R}^{3} \text { such that } f_{0}(x, v) \neq 0\right\} .
$$

Also, if $(f, E, B)$ is a solution of (RVM) with data (RIC), we define for $t>0$,

$$
\begin{gathered}
P(t)=\sup \left\{|v|: \text { there exists } x \in \mathbb{R}^{3}\right. \text { and } \\
\tau \in[0, t] \text { such that } f(x, v, \tau) \neq 0\}+1 .
\end{gathered}
$$

We also define the characteristic curves $(\chi(x, v, t, s), v(x, v, t, s))$ by

$$
\begin{array}{ll}
\frac{d}{d s} \chi=\hat{v}=\left(1+c^{-2} v^{2}\right) v & \chi(x, v, t, t)=x \\
\frac{d}{d s} v=E(\chi, s)+c^{-1} \hat{v} \times B(\chi, s) & v(x, v, t, t)=v .
\end{array}
$$

Note that $(d / d s)[f(\chi, v, s)]=0$ and conclude that $f$ remains non-negative if $f_{0}$ is and that

$$
\sup \left\{f(x, v, t): x \in \mathbb{R}^{3}, v \in \mathbb{R}^{3}\right\}=\left\|f_{0}\right\|_{\infty}
$$


Note also that $f(x, v, t)=0$ if $|x| \geqq S_{0}+t P(t)$.

The following lemma will be used in the proofs of both theorems.

Lemma 1. Let $g$ be a continuous function of compact support on $\mathbb{R}^{3}$, then there exists a constant $D>0$ such that

$$
\eta \int_{|\omega|=1}|g(x+\eta \omega)| d \omega \leqq D
$$

for all $\eta \geqq 0$.

Proof. Note first that

$$
\eta \int_{|\omega|=1}|g(x+\eta \omega)| d \omega \leqq 4 \pi\|g\|_{\infty} \eta
$$

Choose $S$ sufficiently large that $g(x)=0$ if $|x| \geqq S$. Now

$$
\eta \int_{|\omega|=1}|g(x+\eta \omega)| d \omega \leqq \eta\|g\|_{\infty} \int_{|\omega|=1 \text { and }|x+\eta \omega|<S} d \omega .
$$

If $\theta$ denotes a polar angle on the unit sphere with the vector $-x$, then a little analytic geometry shows that if $\eta>S$ then the set $|\omega|=1$ and $|x+\eta \omega|<S$ is contained in the set $\theta \in\left[0, \sin ^{-1}\left(S \eta^{-1}\right)\right]$. Hence for $\eta>S$

$$
\begin{aligned}
& \eta \int_{|\omega|=1}|g(x+\eta \omega)| d \omega \leqq \eta\|g\|_{\infty} \int_{0}^{\sin ^{-1}\left(S \eta^{-1}\right)} 2 \pi \sin \theta d \theta \\
& \quad=2 \pi \eta\|g\|_{\infty}\left(1-\left[\eta^{2}-S^{2}\right]^{1 / 2} \eta^{-1}\right) \\
& \quad=2 \pi\|g\|_{\infty} S^{2}\left(\eta+\left[\eta^{2}-S^{2}\right]^{1 / 2}\right)^{-1}<2 \pi\|g\|_{\infty} S^{2} \eta^{-1} .
\end{aligned}
$$

The lemma follows from (9) and (10).

As in the lemma, D denotes a generic constant which is independent of $c$.

In the appendix an integral representation for $E$ and $B$ is derived as in [6]. In [6] one term of the representation is suppressed since it depends only on the data and hence plays no role in the existence theory. However, this same term plays an essential role in the proof of Theorem 2, so rather than scaling the representation as stated in [6], the entire representation is computed in the appendix.

\section{The Proof of Theorem 1}

To establish Theorem 1 we will apply a theorem from [6] which states that an a priori bound on the $v$ support of $f$ for $0 \leqq t<T$ implies existence of a unique $C^{1}$ solution of $(\mathrm{RVM})$ for $0 \leqq t<T$. Actually [6] treats the case $c=1$ but the generalization to $c \neq 1$ follows immediately by scaling, i.e. the observation that if $(f, E, B)$ is a solution of (RVM) with $c \neq 1$, then

$$
\begin{aligned}
& \bar{f}(x, t)=f\left(\gamma^{-1} x, \gamma^{2} v, \gamma^{-3} t\right), \\
& \bar{E}(x, t)=\gamma^{-5} E\left(\gamma^{-1} x, \gamma^{-3} t\right), \\
& \bar{B}(x, t)=\gamma^{-5} B\left(\gamma^{-1} x, \gamma^{-3} t\right),
\end{aligned}
$$

where $\gamma=c^{1 / 2}$, is a solution of (RVM) with the speed of light normalized to unity. 
Thus we will obtain an a priori bound on $P(t)$ (defined in (6)) independent of $c$ and hence establish existence on a time interval independent of $c$. The uniqueness assertion follows directly from the theorem from [6].

Let $(f, E, B)$ be a $C^{1}$ solution of $(\mathrm{RVM})$ with $c \geqq 1$ and data as in Theorem 1 . Write

$$
E=\mathscr{E}-\mathrm{II}-\mathrm{III}-\mathrm{IV},
$$

where II, III, and IV are the last three terms of (A13) and $\mathscr{E}$ is defined in (A3). Note that

$$
|\hat{v}|=\left(1+c^{-2} v^{2}\right)^{-1 / 2}|v| \leqq c,
$$

and also for $|v| \leqq P$, with $P \geqq 1$,

$$
\begin{aligned}
1+c^{-1} \hat{v} \cdot \omega=1+\left(c^{2}+v^{2}\right)^{-1 / 2} v \cdot \omega & \geqq 1-\left(c^{2}+v^{2}\right)^{-1 / 2}|v| \geqq 1-\left(c^{2}+P^{2}\right)^{-1 / 2} P \\
& =c^{2}\left(c^{2}+P^{2}+P\left[c^{2}+P^{2}\right]^{1 / 2}\right)^{-1} \\
& \geqq c^{2}\left(2\left[c^{2}+P^{2}\right]\right)^{-1},
\end{aligned}
$$

and hence

$$
\left(1+c^{-1} \hat{v} \cdot \omega\right)^{-1} \leqq c^{-2} 2\left(c^{2}+P^{2}\right) \leqq 4 P^{2}
$$

Define

$$
K(t)=\sup \left\{|E(x, t)|+|B(x, t)|: x \in \mathbb{R}^{3}\right\},
$$

and note that by (12)

$$
\left|E(x, t)+c^{-1} \hat{v} \times B(x, t)\right| \leqq|E(x, t)|+|B(x, t)| \leqq K(t) .
$$

Now we use (12) through (15) to estimate the terms of (11). To estimate IV note that

$$
\begin{aligned}
& \left|\left(1+c^{-1} \hat{v} \cdot \omega\right)\left(E+c^{-1} \hat{v} \times B\right)+c^{-2}(\hat{v} \cdot \omega \omega-\hat{v}) \hat{v} \cdot E-\left(\omega+c^{-1} \hat{v}\right) \omega \cdot\left(E+c^{-1} \hat{v} \times B\right)\right| \\
& \quad \leqq\left(1+c^{-1}|\hat{v}|\right) K(t)+c^{-2} 2 \hat{v}^{2} K(t)+\left(1+c^{-1}|\hat{v}|\right) K(t) \leqq 6 K(t),
\end{aligned}
$$

and hence by (13)

$$
\begin{aligned}
|\mathrm{IV}| & \leqq c^{-2} \int_{|x-y|<c t} \int \frac{f\left(y, v, t-c^{-1}|x-y|\right) 6 K\left(t-c^{-1}|x-y|\right)}{|x-y|\left(1+c^{-1} \hat{v} \cdot \omega\right)^{2}\left(1+c^{-2} v^{2}\right)^{1 / 2}} d v d y \\
& \leqq 6 c^{-2}\left(4 P^{2}(t)\right)^{2} \int_{|x-y|<c t} \int \frac{f\left(y, v, t-c^{-1}|x-y|\right) K\left(t-c^{-1}|x-y|\right)}{|x-y|} d v d y .
\end{aligned}
$$

Taking $\tau=t-c^{-1}|x-y|$ we have

$$
\begin{aligned}
\int_{|x-y|<c t} & \int f\left(y, v, t-c^{-1}|x-y|\right)|x-y|^{-1} K\left(t-c^{-1}|x-y|\right) d v d y \\
& =\int_{0|x-y|=c(t-\tau)}^{t} \int_{|v| \leqq P(t)} f(y, v, \tau)|x-y|^{-1} K(\tau) d v d S_{y} c d \tau \\
& \leqq \frac{4 \pi}{3} P^{3}(t)\left\|f_{0}\right\|_{\infty} \int_{0}^{t} \int_{|x-y|=c(t-\tau)}|x-y|^{-1} K(\tau) d S_{y} c d \tau
\end{aligned}
$$




$$
\begin{aligned}
& =\frac{4 \pi}{3} P^{3}(t)\left\|f_{0}\right\|_{\infty} \int_{0}^{t} 4 \pi c(t-\tau) K(\tau) c d \tau \\
& \leqq D c^{2} P^{3}(t) t \int_{0}^{t} K(\tau) d \tau
\end{aligned}
$$

and hence by (16)

$$
|\mathrm{IV}| \leqq D t P^{7}(t) \int_{0}^{t} K(\tau) d \tau
$$

In the same fashion observe that

$$
\begin{aligned}
|\mathrm{III}| & \leqq \int_{|x-v|<c t} \int \frac{f\left(y, v, t-c^{-1}|x-y|\right)\left(1-c^{-2} \hat{v}^{2}\right)\left|\omega+c^{-1} \hat{v}\right|}{|x-y|^{2}\left(1+c^{-1} \hat{v} \cdot \omega\right)^{2}} d v d y \\
& \leqq \int_{|y|<S_{0}+t P(t)} \int_{|v|<P(t)} \frac{\left\|f_{0}\right\|_{\infty}\left(4 P^{2}(t)\right)^{2}\left(1+c^{-1}|\hat{v}|\right)}{|x-y|^{2}} d v d y \\
& \leqq 32\left\|f_{0}\right\|_{\infty} P^{4}(t) \frac{4 \pi}{3} p^{3}(t) \int_{|y|<s_{0}+t P(t)}|x-y|^{-2} d y \\
& \leqq D P^{7}(t) \int_{|y|<S_{0}+t P(t)}|y|^{-2} d y=D P^{7}(t)\left(S_{0}+t P(t)\right) .
\end{aligned}
$$

To estimate II note that for $|v| \leqq P(0)$,

$$
\left(1+c^{-1} \hat{v} \cdot \omega\right)^{-1}\left|\omega-c^{-2} \hat{v} \cdot \omega \hat{v}\right| \leqq 4 P^{2}(0)\left(1+c^{-2} \hat{v}^{2}\right) \leqq 8 P^{2}(0),
$$

and that

$$
(c t)^{-1} \int_{|x-y|=c t} \int f_{0}(y, v) d v d S_{y}=c t \int_{|\omega|=1} \int f_{0}(x+c t \omega, v) d v d \omega \leqq D,
$$

by Lemma 1 . Therefore

$$
|\mathrm{II}| \leqq D \text {. }
$$

To estimate $\mathscr{E}$ we use the following fact which is from Theorem 1 of [12]: Let

$$
u(x, t)=\partial_{t}\left(\frac{t}{4 \pi} \int_{|\omega|=1} \varphi(x+t \omega) d \omega\right)+\frac{t}{\pi} \int_{|\omega|=1} \psi(x+t \omega) d \omega
$$

where $\varphi \in C_{0}^{3}\left(\mathbb{R}^{3}\right)$ and $\psi \in C_{0}^{2}\left(\mathbb{R}^{3}\right)$, then

$$
\sup _{x}|u(x, t)| \leqq D t^{-1}\left(\|\varphi\|_{W^{2,1}}+\|\psi\|_{W^{1,1}}\right)
$$

for $t \geqq 1$. So for all $x \in \mathbb{R}^{3}$ and $t \geqq 0$,

$$
|u(x, t)| \leqq D\left(\|\varphi\|_{W^{1, \infty}}+\|\psi\|_{W^{0, \infty}}+\|\varphi\|_{W^{2,1}}+\|\psi\|_{W^{1,1}}\right) .
$$

Now by approximation, the above inequality holds for $\varphi \in C^{2}\left(\mathbb{R}^{3}\right) \cap W^{1, \infty}\left(\mathbb{R}^{3}\right) \cap W^{2,1}\left(\mathbb{R}^{3}\right)$ and $\psi \in C^{1}\left(\mathbb{R}^{3}\right) \cap W^{0, \infty}\left(\mathbb{R}^{3}\right) \cap W^{1,1}\left(\mathbb{R}^{3}\right)$. Note that by (A3),

$$
\mathscr{E}\left(x, c^{-1} t\right)=\partial_{t}\left(\frac{t}{4 \pi} \int_{|\omega|=1} E_{0}(x+t \omega) d \omega\right)+\frac{t}{4 \pi} \int_{|\omega|=1} c^{-1} \partial_{t} E(x+t \omega, 0) d \omega
$$


and $c^{-1} \partial_{t} E(x, 0)=\nabla \times B_{0}-4 \pi c^{-1} \int f_{0} \hat{v} d v$. Now by the assumptions placed on $f_{0}$, $E_{0}$, and $B_{0}$, we have for all $x$ and $t \geqq 0$,

$$
\begin{aligned}
\left|\mathscr{E}\left(x, c^{-1} t\right)\right| \leqq & D\left(\left\|E_{0}\right\|_{W^{1, \infty}}+\left\|E_{0}\right\|_{W^{2,1}}+\left\|\nabla \times B_{0}\right\|_{W^{0, \infty}}\right. \\
& \left.+\left\|\nabla \times B_{0}\right\|_{W^{1,1}}+c^{-1}\left\|\int f_{0} \hat{v} d v\right\|_{W^{0, \infty}}+c^{-1}\left\|\int f_{0} \hat{v} d v\right\|_{W^{1,1}}\right)
\end{aligned}
$$

Therefore for $c \geqq 1$,

$$
|\mathscr{E}| \leqq D
$$

Just as before we may write

$$
B=\mathscr{B}+\mathrm{II}^{\prime}+\mathrm{III}^{\prime}+\mathrm{IV}^{\prime},
$$

where $\mathscr{B}, \mathrm{II}^{\prime}$, and $\mathrm{III}^{\prime}$, and $\mathrm{IV}^{\prime}$ are the terms of (A14). Since the estimates of these terms are so similar to the previous ones we merely state that

$$
\begin{aligned}
|\mathrm{IV}| & \leqq D t P^{7}(t) \int_{0}^{t} K(\tau) d \tau, \\
|\mathrm{III}| & \leqq D P^{7}(t)\left(S_{0}+t P(t)\right), \\
|\mathrm{II}| & \leqq D, \\
|\mathscr{B}| & \leqq D,
\end{aligned}
$$

as before. Collecting these estimates we have

$$
|E(x, t)|+|B(x, t)| \leqq D+D P^{7}(t)\left(S_{0}+t P(t)\right)+D t P^{7}(t) \int_{0}^{t} K(\tau) d \tau .
$$

Therefore by definition (14) we have

$$
K(t) \leqq D+D P^{7}(t)\left(S_{0}+t P(t)\right)+D t P^{7}(t) \int_{0}^{t} K(\tau) d \tau .
$$

By definition (6) $P$ is nondecreasing so for any $t>0$ and $s \in[0, t]$,

$$
K(s) \leqq D+D P^{7}(t)\left(S_{0}+t P(t)\right)+D t P^{7}(t) \int_{0}^{s} K(\tau) d \tau .
$$

Therefore by Gronwall's inequality

$$
K(s) \leqq\left(D+D P^{7}(t)\left[S_{0}+t P(t)\right]\right) \exp \left(D t P^{7}(t) s\right)
$$

for $0 \leqq s \leqq t$, and in particular

$$
K(t) \leqq\left(D+D P^{7}(t)\left[S_{0}+t P(t)\right]\right) \exp \left(D t^{2} P^{7}(t)\right) .
$$

Now to estimate $P(t)$ choose $(x, v, t)$ such that $f(x, v, t) \neq 0$. Then with $\chi$ and $v$ as defined in (7) we have

$$
\left.f(\chi, v, s)\right|_{s=0}=\left.f(\chi, v, s)\right|_{s=t}=f(x, v, t) \neq 0,
$$

and so

$$
|v(x, v, t, 0)| \leqq P(0)-1
$$


Also by (7) and (14)

$$
\left|\frac{d}{d s} v\right|=\left|E(\chi, s)+c^{-1} \hat{v} \times B(\chi, s)\right| \leqq|E(\chi, s)|+|B(\chi, s)| \leqq K(s),
$$

so

$$
|v|=|v(x, v, t, t)| \leqq|v(x, v, t, 0)|+\int_{0}^{t} K(s) d s \leqq P(0)-1+\int_{0}^{t} K(s) d s .
$$

Now (23) holds for all $v \in \mathbb{R}^{3}$ such that there exists $x \in \mathbb{R}^{3}$ and $t \geqq 0$ with $f(x, v, t) \neq 0$, so by definition of $P,(6)$,

$$
P(t) \leqq P(0)+\int_{0}^{t} K(s) d s
$$

By (22)

$$
P(t) \leqq P(0)+\int_{0}^{t}\left(D+D P^{7}(s)\left[S_{0}+s P(s)\right]\right) \exp \left(D s^{2} P^{7}(s)\right) d s .
$$

Both (22) and (23) hold for all $c \geqq 1$ so (24) does too. Therefore Gronwall's inequality implies $P(t)$ remains finite on some time interval $[0, T)$, where $T>0$ does not depend on $c$.

Now for each $c \geqq 1$ we apply Theorem 1 of [6] to the system (RVM). We note that the a priori bound established above for $P(t)$ must hold for each of the iterates defined in [6]. Thus the iterates defined in [6] converge to a $C^{1}$ solution, $\left(f^{c}, E^{c}, B^{c}\right)$, of (RVP) with data (RIC) on the time interval [0,T), with $T$ as above. Furthermore the solution satisfies (22) and (24). Finally note that since the constants in (22) and (24) are independent of $c$, the functions

$$
\begin{gathered}
\bar{P}(t)=\sup \left\{|v|: \text { there exists } x \in \mathbb{R}^{3},\right. \\
\tau \in[0, t], \text { and } c \geqq 1 \text { such that } \\
\left.f^{c}(x, v, \tau) \neq 0\right\} \\
\bar{K}(t)=\sup \left\{\left|E^{c}(x, t)\right|+\left|B^{c}(x, t)\right|: x \in \mathbb{R}^{3}, c \geqq 1, \text { and } 0 \leqq \tau \leqq t\right\}
\end{gathered}
$$

remain finite on $[0, T)$. This completes the proof of Theorem 1 .

\section{The Proof of Theorem 2}

For notational convenience we'll write $f=f^{c}, E=E^{c}$, and $B=B^{c}$. The heart of the proof consists of comparing the integral representation of $E$ given by (A13) with that of $E^{\infty}$ given in (VP). As before we write

$$
E=\mathscr{E}-\mathrm{II}-\mathrm{III}-\mathrm{IV},
$$

where $\mathscr{E}$, II, III, and IV are the terms of (A13). It is easily seen that for a fixed positive value of $t$

$$
-\mathrm{III} \rightarrow \iint f(y, v, t)|x-y|^{-3}(x-y) d v d y
$$


as $c \rightarrow \infty$. Now since

$$
E^{\infty}=\iint f^{\infty}(y, v, t)|x-y|^{-3}(x-y) d v d y,
$$

we may estimate $E^{\infty}-\left(-\right.$ III) in terms of $f^{\infty}-f$ only. However the convergence in (25) is not uniform in $t$. To attain uniform convergence we will combine the dominant terms of $\mathscr{E}$, II, and III.

Fix $T^{\prime} \in(0, T)$. We will write

$$
A(x, v, t)=B(x, v, t)+O\left(c^{-1}\right)
$$

if there exists a constant $D$ such that

$$
|A(x, v, t)-B(x, v, t)| \leqq D c^{-1}
$$

for all $x \in \mathbb{R}^{3}, v \in \mathbb{R}^{3}, t \in\left[0, T^{\prime}\right]$, and $c \geqq 1$. Note that for $|v| \leqq P(t)$,

$$
|\hat{v}| \leqq\left(1+c^{-2} P^{2}(t)\right)^{-1 / 2} P(t) \leqq P(t),
$$

and recall

$$
\left(1+c^{-1} \hat{v} \cdot \omega\right)^{-1} \leqq 4 P^{2}(t) .
$$

To isolate the dominant term of $\mathscr{E}$ note that $B_{0}=0$, so $\partial_{t} E(x, 0)=$ $-4 \pi \int f_{0} \hat{v} d v$, and hence by Lemma 1 ,

$$
\left|\frac{t}{4 \pi} \int_{|\omega|=1} \partial_{t} E(x+c t \omega, 0) d \omega\right|=c^{-1}\left|c t \int_{|\omega|=1} \int f_{0}(x+c t \omega, v) \hat{v} d v d \omega\right| \leqq D c^{-1} .
$$

Therefore by (A3)

$$
\mathscr{E}=\partial_{t}\left(\frac{t}{4 \pi} \int_{|\omega|=1} E_{0}(x+c t \omega) d \omega\right)+O\left(c^{-1}\right) .
$$

To establish the leading term of II, note that by (26), (13), and Lemma 1 ,

$$
\begin{aligned}
& \left|(c t)^{-1} \int_{|x-y|=c t} \int\left(1+c^{-1} \hat{v} \cdot \omega\right)^{-1} c^{-2} \hat{v} \cdot \omega \hat{v} f_{0}(y, v) d v d S_{y}\right| \\
& \quad \leqq c t \int_{|\omega|=1} 4 P^{2}(0) c^{-2} P^{2}(0) \int f_{0}(x+c t \omega, v) d v d \omega \\
& \quad \leqq 4 P^{4}(0) c^{-2} D=O\left(c^{-2}\right) .
\end{aligned}
$$

Therefore from (A13)

$$
\begin{aligned}
\mathrm{II} & =(c t)^{-1} \int_{|x-y|=c t} \int\left(1+c^{-1} \hat{v} \cdot \omega\right)^{-1} \omega f_{0}(y, v) d v d S_{y}+O\left(c^{-2}\right) \\
& =(c t)^{-1} \int_{|x-y|=c t} \int f_{0}(y, v) d v \omega d S_{y}+O\left(c^{-1}\right)
\end{aligned}
$$

by the following: For $|v| \leqq P(0)$,

$$
\begin{aligned}
\left|\left(1+c^{-1} \hat{v} \cdot \omega\right)^{-1}-1\right| & =\left(1+c^{-1} \hat{v} \cdot \omega\right)^{-1}\left|1-\left(1+c^{-1} \hat{v} \cdot \omega\right)\right| \\
& \leqq 4 P^{2}(0) c^{-1} P(0) .
\end{aligned}
$$


To identify the leading term of III note that since $P$ is nondecreasing

$$
\begin{aligned}
& \left|\int_{|x-y|<c t} \int f\left(y, v, t-c^{-1}|x-y|\right)\right| x-\left.y\right|^{-2}\left(1+c^{-1} \hat{v} \cdot \omega\right)^{-2}\left(c^{-2} \hat{v}^{2}\right)\left(\omega+c^{-1} \hat{v}\right) d v d y \mid \\
& \quad \leqq \int_{|y|<S_{0}+P(t)} \int_{|v|<P(t)}\left\|f_{0}\right\|_{\infty}|x-y|^{-2}\left(4 P^{2}(t)\right)^{2} c^{-2} P^{2}(t)\left(1+c^{-1} P(t)\right) d v d y \\
& \leqq D c^{-2} P^{6}(t)\left(1+c^{-1} P(t)\right) \frac{4 \pi}{3} P^{3}(t) \int_{|y|<S_{0}+t P(t)}|y|^{-2} d y \\
& =D c^{-2} P^{9}(t)\left(1+c^{-1} P(t)\right) 4 \pi\left(S_{0}+t P(t)\right) \leqq D c^{-2}
\end{aligned}
$$

for $t \leqq T^{\prime}$. In the same manner

$$
\left|\int_{|x-y|<c t} \int f\left(y, v, t-c^{-1}|x-y|\right)\right| x-\left.y\right|^{-2}\left(1+c^{-1} \hat{v} \cdot \omega\right)^{-2} c^{-1} \hat{v} d v d y \mid \leqq D c^{-1},
$$

and so by (A13)

$$
\begin{aligned}
\mathrm{III} & =\int_{|x-y|<c t}|x-y|^{-2} \int f\left(y, v, t-c^{-1}|x-y|\right)\left(1+c^{-1} \hat{v} \cdot \omega\right)^{-2} \omega d v d y+O\left(c^{-1}\right) \\
& =\int_{|x-y|<c t}|x-y|^{-2} \int f\left(y, v, t-c^{-1}|x-y|\right) \omega d v d y+O\left(c^{-1}\right)
\end{aligned}
$$

by the following: For $|v| \leqq P(t)$ and $t \leqq T^{\prime}$,

$$
\begin{aligned}
\left|\left(1+c^{-1} \hat{v} \cdot \omega\right)^{-2}-1\right| & =\left(1+c^{-1} \hat{v} \cdot \omega\right)^{-2}\left|1-\left(1+2 c^{-1} \hat{v} \cdot \omega+c^{-2}[\hat{v} \cdot \omega]^{2}\right)\right| \\
& \leqq\left(4 P^{2}(t)\right)^{2} c^{-1}\left(2 P(t)+c^{-1} P^{2}(t)\right) \leqq D c^{-1}
\end{aligned}
$$

To recognize how to combine the leading terms of $\mathscr{E}$, II, and III we will use the following lemma.

Lemma 2. Let $h \in C^{2}\left(\mathbb{R}^{3}\right)$. Assume $\Delta h$ has compact support. Then for $c>0$ and $t \geqq 0$,

$$
\partial_{t}\left(t \int_{|\omega|=1} h(x+c t \omega) d \omega\right)=-\int_{|x-y|>c t}|x-y|^{-1} \Delta h(y) d y .
$$

Proof. Using the fact that $t \int_{|\omega|=1} h(x+c t \omega) d \omega$ is a solution of the homogeneous wave equation, we see that

$$
\partial_{t}^{2}\left(t \int_{|\omega|=1} h(x+c t \omega) d \omega\right)=c^{2} \Delta\left(t \int_{|\omega|=1} h(x+c t \omega) d \omega\right)=c^{2} t \int_{|\omega|=1} \Delta h(x+c t \omega) d \omega .
$$

Substituting $y=x+c t \omega$ yields

$$
\partial_{t}^{2}\left(t \int_{|\omega|=1} h(x+c t \omega) d \omega\right)=t^{-1} \int_{|x-y|=c t} \Delta h(y) d S_{y} .
$$

But note that for $t>0$,

$$
\begin{aligned}
-\partial_{t}\left(\int_{|x-y|>c t}|x-y|^{-1} \Delta h(y) d y\right) & =-\partial_{t}\left(\int_{c t}^{\infty} \int_{|x-y|=r} r^{-1} \Delta h(y) d S_{y} d r\right) \\
& =c \int_{|x-y|=c t}(c t)^{-1} \Delta h(y) d S_{y} \\
& =t^{-1} \int_{|x-y|=c t} \Delta h(y) d S_{y}=\partial_{t}^{2}\left(t \int_{|\omega|=1} h(x+c t \omega) d \omega\right) .
\end{aligned}
$$


Therefore

$$
\partial_{t}\left(t \int_{|\omega|=1} h(x+c t \omega) d \omega\right)+\int_{|x-y|>c t}|x-y|^{-1} \Delta h(y) d y
$$

is a function of $x$ only. But both terms tend to zero as $t$ tends to infinity (using Theorem 1 of [12] for the first term), so

$$
\partial_{t}\left(t \int_{|\omega|=1} h(x+c t \omega) d \omega\right)+\int_{|x-y|>c t}|x-y|^{-1} \Delta h(y) d y=0
$$

which completes the proof of the lemma.

Now in order to rewrite the leading term of $\mathscr{E}, \partial_{t}\left((t / 4 \pi) \int_{|\omega|=1} E_{0}(x+c t \omega) d \omega\right)$, let $h(x)=\iint f_{0}(y, v)|x-y|^{-1} d v d y$ and note that $\nabla h=-E_{0}$ and that $\Delta h=-4 \pi \int f_{0} d v$. Hence by the lemma,

$$
\begin{aligned}
& \partial_{t}\left(\frac{t}{4 \pi} \int_{|\omega|=1} E_{0}(x+c t \omega) d \omega\right)=-\partial_{t}\left(\frac{t}{4 \pi} \int_{|\omega|=1} \nabla h(x+c t \omega) d \omega\right) \\
& \quad=\frac{-1}{4 \pi} \nabla\left(\partial_{t}\left(t \int_{|\omega|=1} h(x+c t \omega) d \omega\right)\right)=\frac{-1}{4 \pi} \nabla\left(-\int_{|x-y|>c t}|x-y|^{-1} \Delta h(y) d y\right) \\
& \quad=-\nabla\left(\int_{|x-y|>c t}|x-y|^{-1} \int f_{0}(y, v) d v d y\right) .
\end{aligned}
$$

Now we substitute $z=x-y$ to get

$$
\begin{aligned}
\partial_{t}\left(\frac{t}{4 \pi} \int_{|\omega|=1} E_{0}(x+c t \omega) d \omega\right) & =-\nabla\left(\int_{|z|>c t}|z|^{-1} \int f_{0}(x-z, v) d v d z\right) \\
& =-\int_{|z|>c t}|z|^{-1} \int \nabla_{x} f_{0}(x-z, v) d v d z \\
& =-\int_{|x-y|>c t}|x-y|^{-1} \int \nabla_{x} f_{0}(y, v) d v d y .
\end{aligned}
$$

Recall that $f_{0}$ has compact support, so by the divergence theorem

$$
\begin{aligned}
& -\int_{|x-y|=c t}|x-y|^{-1} \int f_{0}(y, v) d v \omega_{i} d S_{y}=\int_{|x-y|>c t} \frac{\partial}{\partial y_{i}}\left(|x-y|^{-1} \int f_{0}(y, v) d v\right) d y \\
& =\int_{|x-y|>c t}\left(|x-y|^{-1} \int \frac{\partial f_{0}}{\partial x_{i}}(y, v) d v-|x-y|^{-3}\left(y_{i}-x_{i}\right) \int f_{0}(y, v) d v\right) d y
\end{aligned}
$$

for $i=1,2,3$. Now by (30) we have

$$
\begin{aligned}
\partial_{t}\left(\frac{t}{4 \pi} \int_{|\omega|=1} E_{0}(x+c t \omega) d \omega\right)= & \int_{|x-y|=c t}|x-y|^{-1} \int f_{0}(y, v) d v \omega d S_{y} \\
& -\int_{|x-y|>c t}|x-y|^{-3}(y-x) \int f_{0}(y, v) d v d y \\
= & (c t)^{-1} \int_{|x-y|=c t} \int f_{0}(y, v) d v \omega d S_{y} \\
& -\int_{|x-y|>c t}|x-y|^{-2} \int f_{0}(y, v) d v \omega d y
\end{aligned}
$$


But now if we collect (27), (28), and (29), and use (31) we obtain

$$
\begin{aligned}
\mathscr{E}-\mathrm{II}-\mathrm{III}= & \partial_{t}\left(\frac{t}{4 \pi} \int_{|\omega|=1} E_{0}(x+c t \omega) d \omega\right) \\
& -(c t)^{-1} \int_{|x-y|=c t} \int f_{0}(y, v) d v \omega d S_{y} \\
& -\int_{|x-y|<c t}|x-y|^{-2} \int f\left(y, v, t-c^{-1}|x-y|\right) d v \omega d y+O\left(c^{-1}\right) \\
= & -\int_{|x-y|>c t}|x-y|^{-2} \int f_{0}(y, v) d v \omega d y \\
& -\int_{|x-y|<c t}|x-y|^{-2} \int f\left(y, v, t-c^{-1}|x-y|\right) d v \omega d y+O\left(c^{-1}\right) \\
= & -\int|x-y|^{-2} \int f\left(y, v, \max \left\{0, t-c^{-1}|x-y|\right\}\right) d v \omega d y+O\left(c^{-1}\right) .
\end{aligned}
$$

The remaining term, IV, is of lower order. To bound IV note that with $K(t)$ as defined in (14), we have

$$
K(t) \leqq\left(D+D P^{7}(t)\left[S_{0}+t P(t)\right]\right) \exp \left(D t^{2} P^{7}(t)\right)
$$

so for all $x \in \mathbb{R}^{3}, c \geqq 1$, and $t \in\left[0, T^{\prime}\right]$,

$$
|E(x, t)|+|B(x, t)| \leqq D .
$$

Recalling inequality (16) we have

$$
\begin{aligned}
|\mathrm{IV}| & \leqq D c^{-2} P^{4}(t) \int_{|x-y|<c t} \int f\left(y, v, t-c^{-1}|x-y|\right)|x-y|^{-1} K\left(t-c^{-1}|x-y|\right) d v d y \\
& \leqq D c^{-2} \int_{|y|<S_{0}+t P(t)} \int_{|v|<P(t)}\left\|f_{0}\right\|_{\infty}|x-y|^{-1} d v d y \leqq D c^{-2},
\end{aligned}
$$

for $0 \leqq t \leqq T^{\prime}$. Therefore by (A13) and (32),

$$
E=-\int|x-y|^{-2} \int f\left(y, v, \max \left\{0, t-c^{-1}|x-y|\right\}\right) d v \omega d y+O\left(c^{-1}\right) .
$$

Using the representation of $E^{\infty}$ from (VP), we now estimate

$$
\begin{aligned}
\left|E-E^{\infty}\right|= & \mid O\left(c^{-1}\right) \\
& -\int|x-y|^{-2} \int\left[f\left(y, v, \max \left\{0, t-c^{-1}|x-y|\right\}\right)-f^{\infty}(y, v, t)\right] d v \omega d y \mid \\
\leqq & \int|x-y|^{-2} \int \mid f\left(y, v, \max \left\{0, t-c^{-1}|x-y|\right\}\right) \\
& -f^{\infty}\left(y, v, \max \left\{0, t-c^{-1}|x-y|\right\}\right) \mid d v d y \\
& +\int|x-y|^{-2} \int\left|f^{\infty}\left(y, v, \max \left\{0, t-c^{-1}|x-y|\right\}\right)-f^{\infty}(y, v, t)\right| d v d y+D c^{-1} .
\end{aligned}
$$

Recall that $\left(f^{\infty}, E^{\infty}\right)$ is a $C^{1}$ solution of (VP). Now since $E^{\infty}$ is $C^{1}$ and $f_{0}$ has compact support, it follows that

$$
\begin{aligned}
P^{\infty}(t)= & \sup \left\{|v|: \text { there exists } x \in \mathbb{R}^{3}\right. \\
& \text { and } \left.\tau \in[0, t] \text { such that } f^{\infty}(x, v, \tau) \neq 0\right\}
\end{aligned}
$$

is finite on $[0, T)$. Also $\partial_{t} f^{\infty}$ is bounded on $\mathbb{R}^{6} \times\left[0, T^{\prime}\right]$. Let

$$
Q=P\left(T^{\prime}\right)+P^{\infty}\left(T^{\prime}\right)
$$


and

$$
H(t)=\sup \left\{\left|f(x, v, \tau)-f^{\infty}(x, v, \tau)\right|: x \in \mathbb{R}^{3}, v \in \mathbb{R}^{3} \text {, and } \tau \in[0, t]\right\} .
$$

Then by (34),

$$
\begin{aligned}
\left|E-E^{\infty}\right| \leqq & \int_{|y|<s_{0}+T^{\prime} Q}|x-y|^{-2} \int_{|v|<Q} H\left(\max \left\{0, t-c^{-1}|x-y|\right\}\right) d v d y \\
& \quad+\int_{|y|<s_{0}+T^{\prime} Q}|x-y|^{-2} \int_{|v|<Q} \int_{\max \left\{0, t-c^{-1}|x-y|\right\}}^{t}\left|\partial_{t} f^{\infty}(y, v, \tau)\right| d \tau d v d y+D c^{-1} \\
\leqq & H(t) \int_{|y|<s_{0}+T^{\prime} Q}|x-y|^{-2} \frac{4 \pi}{3} Q^{3} d y \\
& +\int_{|y|<s_{0}+T^{\prime} Q}|x-y|^{-2} \frac{4 \pi}{3} Q^{3} c^{-1}|x-y| D d y+D c^{-1} \\
\leqq & D H(t) \int_{|y|<s_{0}+T^{\prime} Q}|y|^{-2} d y+D c^{-1} \int_{|y| \leqq s_{0}+T^{\prime} Q}|y|^{-1} d y+D c^{-1} \\
\leqq & D H(t)+D c^{-1},
\end{aligned}
$$

for $0 \leqq t \leqq T^{\prime}$.

To estimate $B$ note that $B(x, 0)=0$ and $\partial_{t} B(x, 0)=-c \nabla \times E_{0}=0$, so by (A3)

$$
\mathscr{B}=0 \text {. }
$$

Also, estimating the other terms of (A14),

$$
\begin{aligned}
& \left|(c t)^{-1} \int_{|x-y|=c t} \int\left(1+c^{-1} \hat{v} \cdot \omega\right)^{-1}\left(\omega \times c^{-1} \hat{v}\right) f_{0}(y, v) d v d S_{y}\right| \\
& \quad \leqq c t \int_{|\omega|=1} \int 4 P^{2}(0) c^{-1} P(0) f_{0}(x+c t \omega, v) d v d \omega \leqq 4 P^{3}(0) c^{-1} D
\end{aligned}
$$

by Lemma 1 , and

$$
\begin{aligned}
& \left|c^{-1} \int_{|x-y|<c t} \int f\left(y, v, t-c^{-1}|x-y|\right)\right| x-\left.y\right|^{-2}\left(1+c^{-1} \hat{v} \cdot \omega\right)^{-2}\left(1-c^{-2} \hat{v}^{2}\right)(\omega \times \hat{v}) d v d y \mid \\
& \quad \leqq c^{-1} \int_{|y|<S_{0}+t P(t)} \int_{|v|<P(t)}\left\|f_{0}\right\|_{\infty}|x-y|^{-2}\left(4 P^{2}(t)\right)^{2} P(t) d v d y \\
& \quad \leqq D c^{-1}
\end{aligned}
$$

for $t \leqq T^{\prime}$. The last term of (A14) may be shown to be $O\left(c^{-2}\right)$ in the same manner as the last term of (A13). Thus collecting (37), (38), and (39) we have

$$
B=O\left(c^{-1}\right) \text {. }
$$

We may combine (36) and (40) to get

$$
\left|E+c^{-1} \hat{v} \times B-E^{\infty}\right| \leqq D H(t)+D c^{-1},
$$

for $t \leqq T^{\prime}$, since $|\hat{v}| \leqq c$.

It remains to estimate $f-f^{\infty}$. For notational convenience, define $h=f-f^{\infty}$. The 
estimation of $h$ is via the following equation which follows from (RVM) and (VP):

$$
\begin{aligned}
\partial_{t} h & +\hat{v} \cdot \nabla_{x} h+\left(E+c^{-1} \hat{v} \times B\right) \cdot \nabla_{v} h=\partial_{t} f-\partial_{t} f^{\infty}+\hat{v} \cdot\left(\nabla_{x} f-\nabla_{x} f^{\infty}\right) \\
& +\left(E+c^{-1} \hat{v} \times B\right) \cdot\left(\nabla_{v} f-\nabla_{v} f^{\infty}\right)=-\partial_{t} f^{\infty}-\hat{v} \cdot \nabla_{x} f^{\infty} \\
& -\left(E+c^{-1} \hat{v} \times B\right) \cdot \nabla_{v} f^{\infty}=v \cdot \nabla_{x} f^{\infty}+E^{\infty} \cdot \nabla_{v} f^{\infty}-\hat{v} \cdot \nabla_{x} f^{\infty} \\
& -\left(E+c^{-1} \hat{v} \times B\right) \cdot \nabla_{v} f^{\infty}=(v-\hat{v}) \cdot \nabla_{x} f^{\infty} \\
& +\left(E^{\infty}-E-c^{-1} \hat{v} \times B\right) \cdot \nabla_{v} f^{\infty} .
\end{aligned}
$$

Note that

$$
v-\hat{v}=\left(1+c^{-2} v^{2}\right)^{1 / 2} \hat{v}-\hat{v}=c^{-2}\left(1+\left[1+c^{-2} v^{2}\right]^{1 / 2}\right)^{-1} v^{2} \hat{v}
$$

so (42) becomes

$$
\begin{aligned}
\partial_{t} h+\hat{v} \cdot \nabla_{x} h+\left(E+c^{-1} \hat{v} \times B\right) \cdot \nabla_{v} h= & c^{-2}\left(1+\left[1+c^{-2} v^{2}\right]^{1 / 2}\right)^{-1} v^{2} \hat{v} \cdot \nabla_{x} f^{\infty} \\
& +\left(E^{\infty}-E-c^{-1} \hat{v} \times B\right) \cdot \nabla_{v} f^{\infty} .
\end{aligned}
$$

Note that both $\left|\nabla_{x} f^{\infty}\right|$ and $\left|\nabla_{v} f^{\infty}\right|$ are bounded on $\mathbb{R}^{6} \times\left[0, T^{\prime}\right]$. Also $\nabla_{x} f^{\infty}=0$ if $|v| \geqq P^{\infty}(t)$. Hence by (41)

$$
\begin{aligned}
\left|\partial_{t} h+\hat{v} \cdot \nabla_{x} h+\left(E+c^{-1} \hat{v} \times B\right) \cdot \nabla_{v} h\right| & \leqq D c^{-2}+D\left|E^{\infty}-E-c^{-1} \hat{v} \times B\right| \\
& \leqq D c^{-1}+D H(t),
\end{aligned}
$$

for $0 \leqq t \leqq T^{\prime}$

Next for any $x \in \mathbb{R}^{3}, v \in \mathbb{R}^{3}$, and $t \in\left[0, T^{\prime}\right]$ we may define $\chi$ and $v$ as in (7) and compute (using (43))

$$
\left|\frac{d}{d s} h(\chi, v, s)\right|=\left.\left|\partial_{t} h+\hat{v} \cdot \nabla_{x} h+\left(E+c^{-1} \hat{v} \times B\right) \cdot \nabla_{v} h\right|\right|_{(\chi, v, s)} \leqq D c^{-1}+D H(s),
$$

for $0 \leqq s \leqq T^{\prime}$. Note that

$$
\left.h(\chi, v, s)\right|_{s=0}=\left.f(\chi, v, 0)\right|_{s=0}-\left.f^{\infty}(\chi, v, 0)\right|_{s=0}=\left.f_{0}(\chi, v)\right|_{s=0}-\left.f_{0}(\chi, v)\right|_{s=0}=0,
$$

so

$$
\begin{aligned}
|h(x, v, t)| & =|h(\chi, v, s)|_{s=t}|=| \int_{0}^{t} \frac{d}{d s} h(\chi, v, s) d s \mid \\
& \leqq \int_{0}^{t}\left(D c^{-1}+D H(s)\right) d s \leqq D c^{-1}+D \int_{0}^{t} H(s) d s
\end{aligned}
$$

for $t \leqq T^{\prime}$. But by (35)

$$
H(t)=\sup \left\{|h(x, v, \tau)|: x \in \mathbb{R}^{3}, v \in \mathbb{R}^{3} \text {, and } \tau \in[0, t]\right\},
$$

so it follows from (44) that

$$
H(t) \leqq D c^{-1}+D \int_{0}^{t} H(s) d s
$$

for $0 \leqq t \leqq T^{\prime}$. Now by Gronwall's inequality

$$
H(t) \leqq D c^{-1} \exp (D t) \leqq D c^{-1}
$$


for $0 \leqq t \leqq T^{\prime}$. So by (36) and (40) we have

$$
\begin{aligned}
& \left|f^{c}(x, v, t)-f^{\infty}(x, v, t)\right|+\left|E^{c}(x, t)-E^{\infty}(x, t)\right| \\
& \quad+\left|B^{c}(x, t)\right| \leqq H(t)+D H(t)+D c^{-1} \leqq D c^{-1}
\end{aligned}
$$

for all $x \in \mathbb{R}^{3}, v \in \mathbb{R}, c \geqq 1$, and $t \in\left[0, T^{\prime}\right]$. This completes the proof of Theorem 2 .

\section{Appendix}

Suppose $\rho$ and $j$ are $C^{2}$ functions with compact support in $x$ for each fixed $t$. Suppose also that $E$ and $B$ form a $C^{2}$ solution of Maxwell's equations

$$
\begin{cases}\partial_{t} E=c \nabla \times B-4 \pi j & \nabla \cdot E=4 \pi \rho, \\ \partial_{t} B=-c \nabla \times E & \nabla \cdot B=0 .\end{cases}
$$

Then $E$ and $B$ satisfy

$$
\left\{\begin{array}{l}
\partial_{t}^{2} E-c^{2} \Delta E=-4 \pi\left(c^{2} \nabla \rho+j_{t}\right) \\
\partial_{t}^{2} B-c^{2} \Delta B=4 \pi c \nabla \times j .
\end{array}\right.
$$

Define $\mathscr{E}$ and $\mathscr{B}$ by

$$
\left\{\begin{array}{l}
\mathscr{E}(x, t)=\partial_{t}\left(\frac{t}{4 \pi} \int_{|\omega|=1} E_{0}(x+c t \omega) d \omega\right)+\frac{t}{4 \pi} \int_{|\omega|=1} \partial_{t} E(x+c t \omega, 0) d \omega \\
\mathscr{B}(x, t)=\partial_{t}\left(\frac{t}{4 \pi} \int_{|\omega|=1} B_{0}(x+c t \omega) d \omega\right)+\frac{t}{4 \pi} \int_{|\omega|=1} \partial_{t} B(x+c t \omega, 0) d \omega,
\end{array}\right.
$$

where $\partial_{t} E(x, 0)=c \nabla \times B_{0}-4 \pi \int f_{0} \hat{v} d v$ and $\partial_{t} B(x, 0)=-c \nabla \times E_{0}$. We have

$$
\left\{\begin{array}{l}
E=\mathscr{E}-c^{-2} \int_{|x-y|<c t}|x-y|^{-1}\left(c^{2} \nabla \rho+j_{t}\right)_{\left(y, t-c^{-1}|x-y|\right)} d y \\
B=\mathscr{B}+c^{-1} \int_{|x-y|<c t}|x-y|^{-1}(\nabla \times j)_{\left(y, t-c^{-1}|x-y|\right)} d y .
\end{array}\right.
$$

Now if $(f, E, B)$ is a $C^{1}$ solution of (RVM) with data as in Theorem 1, (A4) may be shown to hold for $\rho=\int f d v$ and $j=\int f \hat{v} d v$ by a standard approximation argument.

Following [6] we define

$$
\left\{\begin{array}{l}
S=\partial_{t}+\hat{v} \cdot \nabla_{x} \\
T=-c^{-1} \omega \partial_{t}+\nabla_{x}
\end{array}\right.
$$

and note that

$$
\left\{\begin{array}{l}
\partial_{t}=\left(1+c^{-1} \hat{v} \cdot \omega\right)^{-1}(S-\hat{v} \cdot T) \\
\nabla_{x}=T+c^{-1}\left(1+c^{-1} \hat{v} \cdot \omega\right)^{-1} \omega(S-\hat{v} \cdot T)
\end{array}\right.
$$

By (A6) we may write

$$
\begin{aligned}
c^{2} \nabla_{x} \rho+\partial_{t} j= & \int\left[\left(1+c^{-1} \hat{v} \cdot \omega\right)^{-1}(c \omega+\hat{v}) S f\right. \\
& \left.+c^{2} T f-\left(1+c^{-1} \hat{v} \cdot \omega\right)^{-1}(c \omega+\hat{v}) \hat{v} \cdot T f\right] d v
\end{aligned}
$$


and hence by (A4)

$$
\begin{aligned}
E= & \mathscr{E}-\left.c^{-2} \int_{|x-y|<c t}|x-y|^{-1} \int\left(c^{2} T f-\left(1+c^{-1} \hat{v} \cdot \omega\right)^{-1}(c \omega+\hat{v}) \hat{v} \cdot T f\right)\right|_{\left(y, v, t-c^{-1}|x-y|\right)} d v d y \\
& -\left.c^{-2} \int_{|x-y|<c t}|x-y|^{-1} \int\left(1+c^{-1} \hat{v} \cdot \omega\right)^{-1}(c \omega+\hat{v}) S f\right|_{\left(y, v, t-c^{-1}|x-y|\right)} d v d y .
\end{aligned}
$$

Note that

$$
\begin{aligned}
\nabla_{y}\left[f\left(y, v, t-c^{-1}|x-y|\right)\right] & =\left(\nabla_{y} f\right)\left(y, v, t-c^{-1}|x-y|\right) \\
& -c^{-1}\left(\partial_{t} f\right)\left(y, v, t-c^{-1}|x-y|\right) \omega \\
& =(T f)\left(y, v, t-c^{-1}|x-y|\right),
\end{aligned}
$$

so we may use the divergence theorem to obtain (for the $i^{\text {th }}$ component)

$$
\begin{aligned}
& \left.\int_{|x-y|<c t}|x-y|^{-1} \int\left[c^{2} T_{i} f-\left(1+c^{-1} \hat{v} \cdot \omega\right)^{-1}(c \omega+\hat{v})_{i} \hat{v} \cdot T f\right]\right|_{\left(y, v, t-c^{-1}|x-y|\right)} d v d y \\
& =-\left.\int_{|x-y|<c t} \int f\left[c^{2} \frac{\partial}{\partial y_{i}}\left(|x-y|^{-1}\right)-\nabla_{y} \cdot\left(\frac{(c \omega+\hat{v})_{i} \hat{v}}{|x-y|\left(1+c^{-1} \hat{v} \cdot \omega\right)}\right)\right]\right|_{(y, v, t-c-1|x-y|)} d v d y \\
& \quad+\int_{|x-y|=c t}|x-y|^{-1} \int\left[c^{2} \omega_{i}-\left(1+c^{-1} \hat{v} \cdot \omega\right)^{-1}(c \omega+\hat{v})_{i} \hat{v} \cdot \omega\right] f(y, v, 0) d v d S_{y} .
\end{aligned}
$$

To compute the derivatives which occur in (A8) note first that $\left(\partial|x-y| / \partial y_{j}\right)=\omega_{j}$ and

$$
\frac{\partial \omega_{i}}{\partial y_{j}}=|x-y|^{-1}\left(\delta_{i j}-\omega_{i} \omega_{j}\right)
$$

Now we compute

$$
\begin{aligned}
& \nabla_{y} \cdot\left(\frac{(c \omega+\hat{v})_{i} \hat{v}}{|x-y|\left(1+c^{-1} \hat{v} \cdot \omega\right)}\right)=\sum_{j=1}^{3} \frac{\partial}{\partial y_{j}}\left(\frac{(c \omega+\hat{v})_{i} \hat{v}_{j}}{|x-y|\left(1+c^{-1} \hat{v} \cdot \omega\right)}\right) \\
&=\sum_{j=1}^{3}|x-y|^{-2}\left(1+c^{-1} \hat{v} \cdot \omega\right)^{-2}\left[|x-y|\left(1+c^{-1} \hat{v} \cdot \omega\right) c|x-y|^{-1}\left(\delta_{i j}-\omega_{i} \omega_{j}\right) \hat{v}_{j}\right. \\
&\left.-(c \omega+\hat{v})_{i} \hat{v}_{j} \frac{\partial}{\partial y_{j}}\left(|x-y|\left(1+c^{-1} \hat{v} \cdot \omega\right)\right)\right] \\
&=|x-y|^{-2}\left(1+c^{-1} \hat{v} \cdot \omega\right)^{-2} \sum_{j=1}^{3}\left[c\left(1+c^{-1} \hat{v} \cdot \omega\right)\left(\delta_{i j}-\omega_{i} \omega_{j}\right) \hat{v}_{j}\right. \\
&-(c \omega+\hat{v})_{i} \hat{v}_{j} \omega_{j}\left(1+c^{-1} \hat{v} \cdot \omega\right) \\
&\left.-(c \omega+\hat{v})_{i} \hat{v}_{j}|x-y| c^{-1} \sum_{k=1}^{3} \hat{v}_{k}|x-y|^{-1}\left(\delta_{j k}-\omega_{j} \omega_{k}\right)\right] \\
&=|x-y|^{-2}\left(1+c^{-1} \hat{v} \cdot \omega\right)^{-2} \sum_{j=1}^{3}\left[( 1 + c ^ { - 1 } \hat { v } \cdot \omega ) \left(c \delta_{i j} \hat{v}_{j}\right.\right. \\
&\left.\left.-2 c \omega_{i} \omega_{j} \hat{v}_{j}-\hat{v}_{i} \hat{v}_{j} \omega_{j}\right)-(c \omega+\hat{v})_{i} \hat{v}_{j} c^{-1}\left(\hat{v}_{j}-\omega \cdot \hat{v} \omega_{j}\right)\right] \\
&=|x-y|^{-2}\left(1+c^{-1} \hat{v} \cdot \omega\right)^{-2}\left[( 1 + c ^ { - 1 } \hat { v } \cdot \omega ) \left(c \hat{v}_{i}-2 c \hat{v} \cdot \omega \omega_{i}\right.\right. \\
&\left.\left.-\hat{v} \cdot \omega \hat{v}_{i}\right)-c^{-1}(c \omega+\hat{v})_{i}\left(\hat{v}^{2}-[\omega \cdot \hat{v}]^{2}\right)\right] \\
&=|x-y|^{-2}\left(1+c^{-1} \hat{v} \cdot \omega\right)^{-2}\left[\left(c-c^{-1} \hat{v}^{2}\right) \hat{v}_{i}-2 c \hat{v} \cdot \omega \omega_{i}-\omega_{i}(\hat{v} \cdot \omega)^{2}-\omega_{i} \hat{v}^{2}\right]
\end{aligned}
$$


and hence

$$
\begin{aligned}
c^{2} & \frac{\partial}{\partial y_{i}}\left(|x-y|^{-1}\right)-\nabla_{y} \cdot\left(\frac{(c \omega+\hat{v})_{i} \hat{v}}{|x-y|\left(1+c^{-1} \hat{v} \cdot \omega\right)}\right) \\
= & |x-y|^{-2}\left(1+c^{-1} \hat{v} \cdot \omega\right)^{-2}\left[-c^{2} \omega_{i}\left(1+c^{-1} \hat{v} \cdot \omega\right)^{2}\right. \\
& \left.-\left(c-c^{-1} \hat{v}^{2}\right) \hat{v}_{i}+2 c \hat{v} \cdot \omega \omega_{i}+\omega_{i}(\hat{v} \cdot \omega)^{2}+\omega_{i} \hat{v}^{2}\right] \\
\quad & |x-y|^{-2}\left(1+c^{-1} \hat{v} \cdot \omega\right)^{-2}\left[-c^{2} \omega_{i}-\left(c-c^{-1} \hat{v}^{2}\right) \hat{v}_{i}+\omega_{i} \hat{v}^{2}\right] \\
& =-|x-y|^{-2}\left(1+c^{-1} \hat{v} \cdot \omega\right)^{-2}\left(c^{2}-\hat{v}^{2}\right)\left(\omega+c^{-1} \hat{v}\right)_{i} .
\end{aligned}
$$

Substituting this result into (A8) yields

$$
\begin{aligned}
& \left.\int_{|x-y|<c t}|x-y|^{-1} \int\left[c^{2} T_{i} f-\left(1+c^{-1} \hat{v} \cdot \omega\right)^{-1}(c \omega+\hat{v})_{t} \hat{v} \cdot T f\right]\right|_{\left(y, v, t-c^{-1}|x-y|\right.} d v d y \\
& =\left.\int_{|x-y|<c t} \int f|x-y|^{-2}\left(1+c^{-1} \hat{v} \cdot \omega\right)^{-2}\left(c^{2}-\hat{v}^{2}\right)\left(\omega+c^{-1} \hat{v}\right)_{i}\right|_{\left(y, v, t-c^{-1}|x-y|\right)} d v d y \\
& \quad+(c t)^{-1} \int_{|x-y|=c t} \int c^{2}\left(1+c^{-1} \hat{v} \cdot \omega\right)^{-1}\left(\omega-c^{-2} \hat{v} \cdot \omega \hat{v}\right)_{i} f_{0}(y, v) d v d S_{y} .
\end{aligned}
$$

The last term of (A10) is the one which was suppressed in [6] and plays a key role in the proof of Theorem 2.

To address the "Sf" term in (A7) note that

$$
S f=\partial_{t} f+\hat{v} \cdot \nabla_{x} f=-\left(E+c^{-1} \hat{v} \times B\right) \cdot \nabla_{v} f=-\nabla_{v} \cdot\left(f\left[E+c^{-1} \hat{v} \times B\right]\right),
$$

since a short computation shows that

$$
\nabla_{v} \cdot\left(E+c^{-1} \hat{v} \times B\right)=0 .
$$

Therefore, by the divergence theorem

$$
\int\left(1+c^{-1} \hat{v} \cdot \omega\right)^{-1}(c \omega+\hat{v})_{i} S f d v=\int f\left[E+c^{-1} \hat{v} \times B\right] \cdot \nabla_{v}\left[\left(1+c^{-1} \hat{v} \cdot \omega\right)^{-1}(c \omega+\hat{v})_{i}\right] d v .
$$

Now we compute

$$
\begin{aligned}
& \frac{\partial}{\partial v_{j}}\left[\left(1+c^{-1} \hat{v} \cdot \omega\right)^{-1}(c \omega+\hat{v})_{i}\right]=\frac{\partial}{\partial v_{j}}\left[\frac{c\left(1+c^{-2} v^{2}\right)^{1 / 2} \omega_{i}+v_{i}}{\left(1+c^{-2} v^{2}\right)^{1 / 2}+c^{-1} v \cdot \omega}\right] \\
&=\left[\left(1+c^{-2} v^{2}\right)^{1 / 2}+c^{-1} v \cdot \omega\right]^{-2}\left[\left(\left[1+c^{-2} v^{2}\right]^{1 / 2}+c^{-1} v \cdot \omega\right)\left(c^{-1} \hat{v}_{j} \omega_{i}+\delta_{i j}\right)\right. \\
&\left.-\left(c\left[1+c^{-2} v^{2}\right]^{1 / 2} \omega_{i}+v_{i}\right)\left(c^{-2} \hat{v}_{j}+c^{-1} \omega_{j}\right)\right] \\
&=\left(1+c^{-2} v^{2}\right)^{-1 / 2}\left(1+c^{-1} \hat{v} \cdot \omega\right)^{-2}\left[\left(1+c^{-1} \hat{v} \cdot \omega\right)\left(c^{-1} \hat{v}_{j} \omega_{i}+\delta_{i j}\right)\right. \\
&\left.-(c \omega+\hat{v})_{i}\left(c^{-2} \hat{v}_{j}+c^{-1} \omega_{j}\right)\right] \\
&=\left(1+c^{-2} v^{2}\right)^{-1 / 2}\left(1+c^{-1} \hat{v} \cdot \omega\right)^{-2}\left[\left(1+c^{-1} \hat{v} \cdot \omega\right) \delta_{i j}\right. \\
&\left.+c^{-2}\left(\hat{v} \cdot \omega \omega_{i}-\hat{v}_{i}\right) \hat{v}_{j}-\left(\omega+c^{-1} \hat{v}\right)_{i} \omega_{j}\right],
\end{aligned}
$$

and hence

$$
\begin{aligned}
&\left(E+c^{-1} \hat{v} \times B\right) \cdot \nabla_{v}\left[\left(1+c^{-1} \hat{v} \cdot \omega\right)^{-1}(c \omega+\hat{v})_{i}\right] \\
&=\left(1+c^{-2} v^{2}\right)^{-1 / 2}\left(1+c^{-1} \hat{v} \cdot \omega\right)^{-2}\left[\left(1+c^{-1} \hat{v} \cdot \omega\right)\left(E+c^{-1} \hat{v} \times B\right)_{i}\right. \\
&+c^{-2}(\hat{v} \cdot \omega \omega-\hat{v})_{i} \hat{v} \cdot E-\left(\omega+c^{-1} \hat{v}_{i} \omega \cdot\left(E+c^{-1} \hat{v} \times B\right)\right] .
\end{aligned}
$$


Substitution into (A11) yields

$$
\begin{aligned}
\int\left(1+c^{-1} \hat{v} \cdot \omega\right)^{-1}(c \omega+\hat{v})_{i} S f d v \\
=\int f\left(1+c^{-2} v^{2}\right)^{-1 / 2}\left(1+c^{-1} \hat{v} \cdot \omega\right)^{-2} \\
\quad \cdot\left[\left(1+c^{-1} \hat{v} \cdot \omega\right)\left(E+c^{-1} \hat{v} \times B\right)_{i}+c^{-2}(\hat{v} \cdot \omega \omega-\hat{v})_{i} \hat{v} \cdot E\right. \\
\left.\quad-\left(\omega+c^{-1} \hat{v}\right)_{i} \omega \cdot\left(E+c^{-1} \hat{v} \times B\right)\right] d v .
\end{aligned}
$$

Finally we obtain the desired representation for $E$ by substituting (A10) and (A12) into (A7):

$$
\begin{aligned}
E= & \mathscr{E}-(c t)^{-1} \int_{|x-y|=c t} \int\left(1+c^{-1} \hat{v} \cdot \omega\right)^{-1}\left(\omega-c^{-2} \hat{v} \cdot \omega \hat{v}\right) f_{0}(y, v) d v d S_{y} \\
& -\left.\int_{|x-y|<c t} \int f|x-y|^{-2}\left(1+c^{-1} \hat{v} \cdot \omega\right)^{-2}\left(1-c^{-2} \hat{v}^{2}\right)\left(\omega+c^{-1} \hat{v}\right)\right|_{\left(y, v, t-c^{-1}|x-y|\right.} d v d y \\
& -c^{-2} \int_{|x-y|<c t} \int f|x-y|^{-1}\left(1+c^{-1} \hat{v} \cdot \omega\right)^{-2}\left(1+c^{-2} v^{2}\right)^{-1 / 2} \\
& \cdot\left[\left(1+c^{-1} \hat{v} \cdot \omega\right)\left(E+c^{-1} \hat{v} \times B\right)\right. \\
& \left.+c^{-2}(\hat{v} \cdot \omega \omega-\hat{v}) \hat{v} \cdot E-\left(\omega+c^{-1} \hat{v}\right) \omega \cdot\left(E+c^{-1} \hat{v} \times B\right)\right]\left.\right|_{\left(y, v, t-c^{-1}|x-y|\right)} d v d y .
\end{aligned}
$$

The representation for $B$ may be computed in the same manner as for $E$. Since the computation is so similar, we only state the result:

$$
\begin{aligned}
B= & \mathscr{B}+(c t)^{-1} \int_{|x-y|=c t} \int\left(1+c^{-1} \hat{v} \cdot \omega\right)^{-1}\left(\omega \times c^{-1} \hat{v}\right) f_{0}(y, v) d v d S_{y} \\
& +\left.c^{-1} \int_{|x-y|<c t} \int f|x-y|^{-2}\left(1+c^{-1} \hat{v} \cdot \omega\right)^{-2}\left(1-c^{-2} \hat{v}^{2}\right)(\omega \times \hat{v})\right|_{\left(y, v, t-c^{-1}|x-y|\right)} d v d y \\
& +c^{-2} \int_{|x-y|<c t} \int f|x-y|^{-1}\left(1+c^{-1} \hat{v} \cdot \omega\right)^{-2}\left(1+c^{-2} v^{2}\right)^{-1 / 2} \\
& \cdot\left[\left(1+c^{-1} \hat{v} \cdot \omega\right) \omega \times\left(E+c^{-1} \hat{v} \times B\right)\right. \\
& \left.-c^{-2}(\omega \times \hat{v})(\hat{v}+c \omega) \cdot\left(E+c^{-1} \hat{v} \times B\right)\right]\left.\right|_{\left(y, v, t-c^{-1}|x-y|\right)} d v d y
\end{aligned}
$$

Acknowledgement. The author acknowledges with pleasure many helpful conversations with Professor Robert Glassey, Indiana University.

\section{References}

1. Arsen'ev, A.: Global existence of a weak solution of Vlasov's system of equations. USSR Comput. Math. Math. Phys. 15, 131-143 (1975)

2. Asano, K., Ukai, S.: On the Vlasov-Poisson limit of the Vlasov-Maxwell equation. Preprint

3. Bardos, C., Degond, P.: Global existence for the Vlasov-Poisson equation in three space variables with small initial data. Preprint

4. Batt, J.: Global symmetric solutions of the initial-value problem of steller dynamics. J. Differ. Equations 25, 342-364 (1977)

5. Degond, P.: Local existence of solutions of the Vlasov-Maxwell equations and convergence to the Vlasov-Poisson equations for infinite light velocity. Internal report no. 117, Centre de Mathématiques appliquées, Ecole polytechnique, Paris 
6. Glassey, R., Strauss, W.: Singularity formation in a collisionless plasma could occur only at high velocities. To appear in Arch. Ration. Mech. Anal.

7. Glassey, R., Schaeffer, J.: On symmetric solutions of the relativistic Vlasov-Poisson system. Commun Math. Phys. 101, 459 (1985)

8. Horst, E.: On the classical solutions of the initial value problem for the unmodified nonlinear Vlasov equation. Parts I and II. Math. Methods Appl. Sci. 3, 229-248 (1981); 4, 19-32 (1982)

9. Horst, E., Hunze, R.: Weak solutions of the initial value problem for the unmodified nonlinear Vlasov-equation. Math. Methods Appl. Sci. 6, 262-279 (1984)

10. Kurth, R.: Das Anfangswertproblem der Stellardynamik. Z. Astrophys. 30, 213-229 (1952)

11. Van Kampen, N. G., Felderhof, B. U.: Theoretical methods in plasma physics. Amsterdam. NorthHolland, 1967 (p. 170)

12. von Wahl, W.: $L^{p}$-Decay rate for homogeneous wave equations. Math. Z. 120, 93-106 (1971)

13. Weibel, E.: L'équation de Vlasov dans la théorie spéciale de la relativite. Plasma Phys. 9, 665-670 (1967)

14. Wollman, S.: The spherically symmetric Vlasov-Poisson system. J. Differ. Equations 35, 30-35 (1980)

15. Wollman, S.: An existence and uniqueness theorem for the Vlasov-Maxwell system. Commun. Pure. Appl. Math. 37, 457-462 (1984)

Communicated by L. Nirenberg

Received August 5, 1985 
